\title{
Effect of baicalein on the expression of SATB1 in human breast cancer cells
}

\author{
XIAO-YAN GAO, XING-HUAN XUE, YI-NAN MA and SHU-QUN ZHANG
}

Department of Oncology, The Second Affiliated Hospital of Xi'an Jiaotong University, Xi'an, Shaanxi 710004, P.R. China

Received May 16, 2014; Accepted January 12, 2015

DOI: $10.3892 /$ etm.2015.2329

\begin{abstract}
The aim of the present study was to investigate the effects of baicalein on the protein expression of SATB1 in the MDA-MB-231 human breast cancer cell line. MDA-MB-231 cells were treated with various concentrations of baicalein $(0,10,20,40 \mu \mathrm{M})$. Following treatment, a 3-(4,5-dimethylthiazol-2-yl)-2,5-diphenyl tetrazolium bromide assay and wound healing assay were used to detect the changes in cell proliferation and migration. In addition, western blot analysis was performed to detect the changes in the protein expression levels of SATB1 in the MDA-MB-231 cell line. With the prolongation of administration time and the increase in drug concentration, the inhibitory effect of baicalein on the proliferation and migration of MDA-MB-231 cells gradually increased in a time- and dose-dependent manner $(\mathrm{P}<0.05)$. In addition, baicalein was shown to markedly decrease the protein expression levels of SATB1 in the MDA-MB-231 cells. With increasing drug concentrations, the protein expression levels of SATB1 decreased gradually $(\mathrm{P}<0.05)$. Therefore, baicalein was demonstrated to inhibit the proliferation of MDA-MB-231 cells and downregulate the protein expression of SATB1, indicating that baicalein can significantly inhibit the proliferation, migration and invasiveness of MDA-MB-231 cells by downregulating the expression of SATB1.
\end{abstract}

\section{Introduction}

Breast cancer is a common malignant tumor in females, which has the second highest cancer mortality rate among females worldwide (1). The morbidity rate is increasing yearly; thus, breast cancer poses as a serious threat to female health. The dissemination and proliferation of tumor cells to other organs is the major factor causing difficulty for clinical treatment, and is also the main cause of mortality in patients with breast cancer. Almost $50 \%$ of breast cancer patients already have

Correspondence to: Dr Shu-Qun Zhang, Department of Oncology, The Second Affiliated Hospital of Xi'an Jiaotong University, 157 Xiwu Road, Xi'an, Shaanxi 710004, P.R. China

E-mail: shuqunzhangn@163.com

Key words: baicalein, SATB1, breast cancer cell line distant metastases $(2,3)$ at their initial diagnosis. Therefore, it is particularly important to prevent and control tumor metastasis in breast cancer.

SATB1 (special AT-rich sequence binding protein 1) is a type of tissue specific nuclear matrix binding protein, which has significant expression in thymus cells, progenitor cells and the epithelial basal layer, with almost no expression in other normal cells and tissues (4-6). As a global chromatin organizer and transcription factor, SATB1 has emerged as a key factor integrating higher-order chromatin architecture with gene regulation (7-9). The protein plays an important role in $\mathrm{T}$ cell development, early erythroid differentiation, cell homeostasis and responses to a number of types of stimulation $(4,5,7,10,11)$.

A previous study reported that SATB1 has an abnormally high expression in a variety of tumor cells, and controls $>1,000$ types of tumor-associated genes, affecting the promotion of tumor growth and metastasis (12). Consequently, SATB1 is regarded as a potentially important target molecule for antitumor drugs.

Baicalein (molecular formula, $\mathrm{C}_{15} \mathrm{H}_{10} \mathrm{O}_{5}$; molecular weight, $270.24 \mathrm{gmol}^{-1}$ ) is a monomer isolated from Scutellaria baicalensis Georgi, which is the main effective component in Radix Scutellariae. Baicalein has been reported to inhibit tumor cell proliferation, invasiveness and metastasis in human breast cancer, liver cancer and pancreatic cancer cell lines (13-15). However, the specific molecular mechanism underlying the inhibition of tumor growth and metastasis is yet to be elucidated. The present study investigated a novel antitumor mechanism of baicalein in the MDA-MB-231 human breast cancer cell line, where baicalein may exert an antitumor, -invasion and -metastasis function by inhibiting the protein expression of SATB1.

\section{Materials and methods}

Cell culture. An MDA-MB-231 cell line (Shanghai Institutes of Biological Sciences, Shanghai, China) was cultured in RPMI-1640 medium (Hyclone; GE Healthcare, Logan, UT, USA), supplemented with $10 \%$ fetal bovine serum (Hyclone; GE Healthcare), in a humidified atmosphere of $5 \% \mathrm{CO}_{2}$ at $37^{\circ} \mathrm{C}$. The medium was changed every other day. Cells were passaged when $80 \%$ of the bottle wall was covered.

3-(4,5-dimethylthiazol-2-yl)-2,5-diphenyl tetrazolium bromide (MTT) assay. MDA-MB-231 cells were seeded in a 
96-well plate at a density of $10^{4}$ cells/well and treated with $200 \mu \mathrm{l}$ baicalein (99\% purity; Sigma-Aldrich, St. Louis, MO, USA) at a concentration of $0,5,10,20,40$ or $80 \mu \mathrm{M}$. The cells were incubated at $37^{\circ} \mathrm{C}$ for 24,48 or $72 \mathrm{~h}$. For the blank control group, $200 \mu \mathrm{l}$ common cell culture fluid was added. Each concentration was set as six parallel holes. Thereafter, the media was changed and the cells were incubated with $20 \mu \mathrm{l}$ MTT (5 mg/ml; Sigma-Aldrich) for $4 \mathrm{~h}$. The mixture was centrifuged at $12,000 \mathrm{xg}$ at $4^{\circ} \mathrm{C}$ for $10 \mathrm{~min}$. The supernatant was removed, 50 ul DMSO was added to each well, and the solutions were agitated on a decolorization shaker (ZHWY-100B; Zhicheng Analytical Instrument Manufacturing Co., Ltd., Shangghai, China) for $10 \mathrm{~min}$. The optical density (OD) of each well was measured using an enzyme immunoassay analyzer (PowerWave XS; BioTek Instruments Inc., Winooski, VT, USA) at $490 \mathrm{~nm}$. The inhibitory rate of cell proliferation (inhibition ratio, IR) was calculated as follows: $\mathrm{IR}=(1$ - mean OD value of the experimental group/mean OD value of the control group) $\mathrm{x} 100 \%$. Using the cell proliferation inhibitory rates with the different concentrations of baicalein at $48 \mathrm{~h}$, the $50 \%$ inhibitory concentration $\left(\mathrm{IC}_{50}\right)$ of baicalein was calculated.

Wound healing assay. MDA-MB-231 cells were seeded in a six-well plate at a density of $2 \times 10^{5}$ cells/well with complete medium overnight to obtain a full confluent monolayer. The cells were scraped away vertically after $24 \mathrm{~h}$ using a plastic tip. Each well was washed three times with phosphate-buffered saline (PBS) to remove the cell debris, and then further incubated for $48 \mathrm{~h}$ in serum-free RPMI-1640 medium with different concentrations of baicalein $(0,10$ and $20 \mu \mathrm{M})$. Two wells were used for each group, and the experiment was repeated three times. The average distance of cell migration to the injured area was determined under a TS100 inverted microscope (magnification, x40; Nikon Corporation, Tokyo, Japan) at 0,24 and $48 \mathrm{~h}$.

Western blot analysis. MDA-MB-231 cells were lysed with radioimmunoprecipitation assay lysis buffer (Beijing Biosynthesis Biotechnology Co., Ltd., Beijing, China), after which the total cellular protein was extracted. The protein concentration in the supernatants was determined using a bicinchoninic acid assay (Beijing Biosynthesis Biotechnology Co., Ltd.) with a Varioskan Flash Multimode microplate spectrophotometer (Thermo Fisher Scientific, Waltham, MA, USA). An equal amount of protein was separated using $10 \%$ SDS-polyacrylamide gel electrophoresis and transferred onto polyvinylidene fluoride (PVDF) membranes $(0.45 \mu \mathrm{m}$; Millipore Corporation, Bedford, MA, USA), according to the semi-dry protein transfer method. The membranes were blocked with 5\% skimmed milk powder for 90 min, and then incubated with primary antibodies against SATB1 (\#ab49061; rabbit polyclonal; 1.25:1,000 dilution; Abcam, Cambridge, MA, USA) and $\beta$-actin (\#sc-47778; rabbit polyclonal; 1:1,000 dilution; \#bs-0295G-HRP; Santa Cruz Biotechnology, Inc., Santa Cruz, CA, USA) for $4^{\circ} \mathrm{C}$ overnight. Subsequently, the membranes were incubated with a horseradish peroxidase-conjugated secondary antibody (1:1,000 dilution; Beijing Biosynthesis Biotechnology Co., Ltd.) at $37^{\circ} \mathrm{C}$ for $1 \mathrm{~h}$. Finally, the PVDF membranes were washed with PBS and

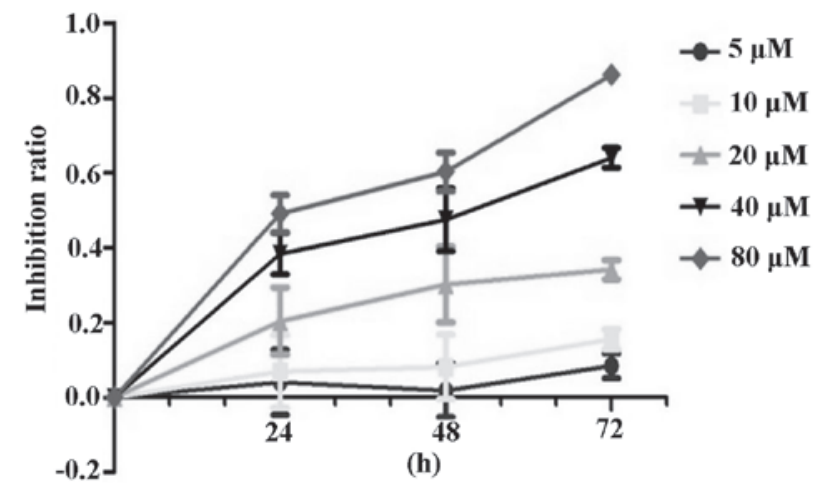

Figure 1. Inhibition of MDA-MB-231 cell proliferation by baicalein in a time- and dose-dependent manner. Results are expressed as the mean \pm standard deviation $(\mathrm{n}=6)$. The inhibition rate of the control group $(0 \mu \mathrm{M})$ was $0 \%$ and therefore the data are not compared here.

colored using enhanced chemiluminescence (Thermo Fisher Scientific), after which detection was performed using a gel imaging system (Syngene, Frederick, MD, USA). The experiment was repeated three times.

Statistical analysis. Data are presented as the mean \pm standard deviation. The data were analyzed using SPSS 18.0 software (SPSS, Inc., Chicago, IL, USA) and GraphPad Prism software (GraphPad Software, Inc., La Jolla, CA, USA). A one-way analysis of variance test was used to compare the differences among groups. The $\mathrm{RxC}$ contingency table and $\chi^{2}$ test were used to compare the quantitative differences among groups. $\mathrm{P}<0.05$ was considered to indicate a statistically significant difference.

\section{Results}

Baicalein suppresses the proliferation of MDA-MB-231 cells. Baicalein administration suppressed the proliferation of MDA-MB-231 cells. When compared with the control group, baicalein administration was shown to significantly inhibit MDA-MB-231 cell proliferation at various concentrations $(5,10,20,40$ and $80 \mu \mathrm{M})$ for 48 and $72 \mathrm{~h}(\mathrm{P}<0.05)$. At $24 \mathrm{~h}$, the inhibition rate increased with increased drug concentration but this was not statistically significant. With the prolongation of administration time and increase in drug concentration, the inhibitory effect of baicalein on the proliferation of MDA-MB-231 cells gradually increased in a time- and dose-dependent manner $(\mathrm{P}<0.05)$, as shown in Table I and Fig. 1 . The $\mathrm{IC}_{50}$ value was determined to be $27.92 \mu \mathrm{M}$ when treated with baicalein for $48 \mathrm{~h}$.

Baicalein affects the migration of MDA-MB-231 cells in vitro. Detection of the cell migration capacity is also a method for analyzing the metastatic potential of tumor cells. Under an inverted microscope, the cell morphology of MDA-MB-231 cells exhibited no marked changes in the control group and $10 \mu \mathrm{M}$ baicalein treatment group. However, in the cells treated with $20 \mu \mathrm{M}$ baicalein for $48 \mathrm{~h}$, the cell morphology had changed from the original long spindle shape to a round shape. As shown in Fig. 2, the number of cells that had migrated into the scratch area in the baicalein intervention group $(20 \mu \mathrm{M})$ was significantly lower when compared with the control group 
Table I. Inhibitory effects of baicalein on MDA-MB-231 cell proliferation.

\begin{tabular}{|c|c|c|c|c|c|c|}
\hline \multirow[b]{2}{*}{ Baicalein $(\mu \mathrm{M})$} & \multicolumn{2}{|c|}{$24 \mathrm{~h}$} & \multicolumn{2}{|c|}{$48 \mathrm{~h}$} & \multicolumn{2}{|c|}{$72 \mathrm{~h}$} \\
\hline & OD & $\operatorname{IR}(\%)$ & OD & $\operatorname{IR}(\%)$ & OD & $\operatorname{IR}(\%)$ \\
\hline 0 & $0.406 \pm 0.031$ & 0 & $0.603 \pm 0.036$ & 0 & $0.981 \pm 0.027$ & 0 \\
\hline 5 & $0.482 \pm 0.013$ & -22.84 & $0.533 \pm 0.051$ & 13.20 & $0.841 \pm 0.063$ & 14.27 \\
\hline 10 & $0.408 \pm 0.052$ & -6.53 & $0.507 \pm 0.033$ & 18.18 & $0.824 \pm 0.025$ & 20.08 \\
\hline 20 & $0.338 \pm 0.029$ & 20.63 & $0.356 \pm 0.025$ & 46.90 & $0.465 \pm 0.011$ & 52.60 \\
\hline 40 & $0.316 \pm 0.016$ & 27.26 & $0.283 \pm 0.022$ & 60.49 & $0.212 \pm 0.030$ & 78.39 \\
\hline 80 & $0.264 \pm 0.014$ & 42.67 & $0.207 \pm 0.023$ & 74.76 & $0.193 \pm 0.014$ & 80.33 \\
\hline
\end{tabular}

Results are expressed as the mean \pm standard deviation $(\mathrm{n}=6)$. OD, optical density; IR, inhibition ratio.

A

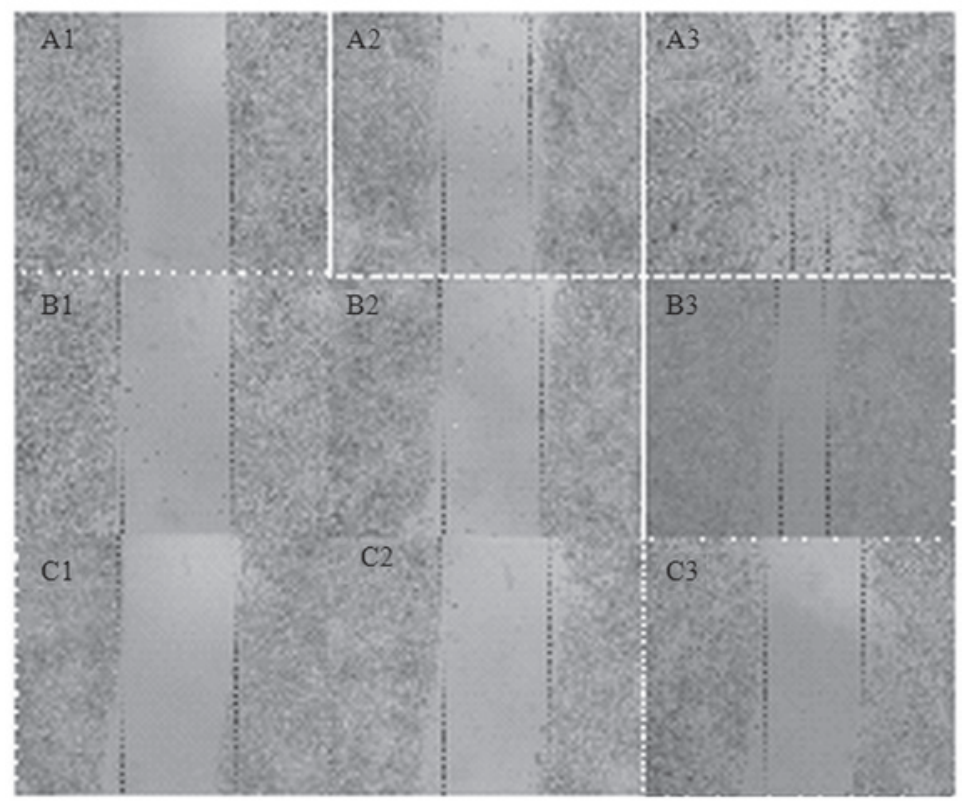

B

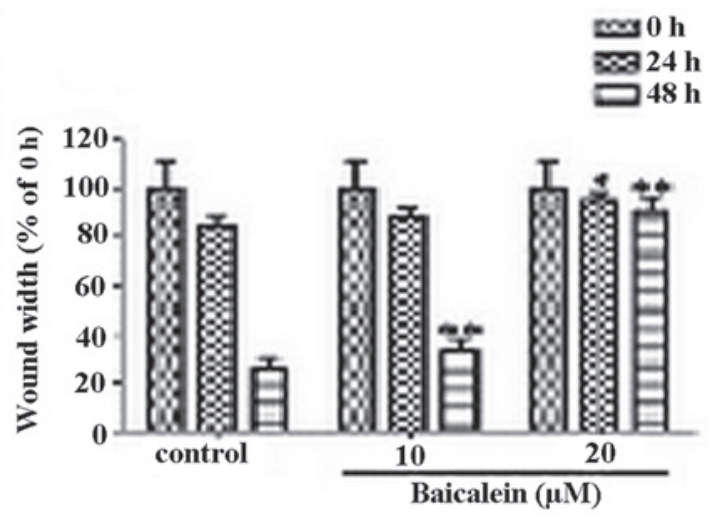

Figure 2. Effect of baicalein on MDA-MB-231 cell migration in vitro. (A) Images of wounded cells treated with baicalein at various concentrations (A1-A3, $0 \mu \mathrm{M}$; B1-B3, $10 \mu \mathrm{M}$; C1-C3, $20 \mu \mathrm{M}$ ) for 0,24 and $48 \mathrm{~h}$, respectively (magnification, $\mathrm{x} 40$ ). (B) Quantification of the wound healing assay (current width/original width). Results are expressed as the mean \pm standard deviation $(\mathrm{n}=6)$. ${ }^{*} \mathrm{P}<0.05$ and ${ }^{* *} \mathrm{P}<0.01$, vs. control (one-way analysis of variance). \% of $0 \mathrm{~h}$, current width (at 24 or $48 \mathrm{~h}$ )/original width (at $0 \mathrm{~h}$ ) x100.

for 24 and $48 \mathrm{~h}(\mathrm{P}<0.05)$. With increasing concentrations of baicalein, the scratch width decreased significantly when compared with the control group at 24 and $48 \mathrm{~h}$ (Fig. 2A); this effect was time- and dose- dependent $(\mathrm{P}<0.05$; Fig. 2B). The inhibition rate was $\sim 10.8$ and $88.7 \%$ at $48 \mathrm{~h}$ with 10 and $20 \mu \mathrm{M}$ baicalein, respectively. The scratch widths of the different groups at each time point are shown in Fig. 2.

Baicalein inhibits SATB1 protein expression in MDA-MB-231 cells. When compared with the control group, the protein 
A

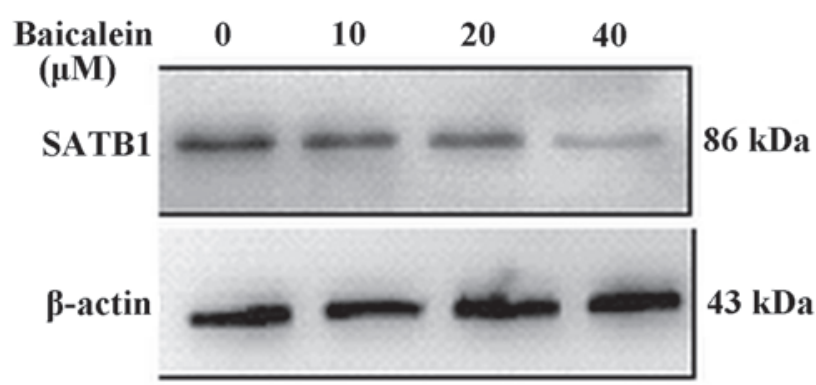

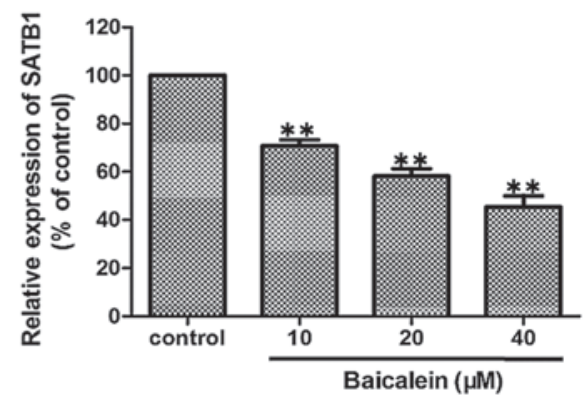

Figure 3. Effect of baicalein on the protein expression of SATB1 in MDA-MB-231 cells. (A) Western blot analysis results indicate the downregulation of SATB1 expression in MDA-MB-231 cells by baicalein in a in dose-dependent manner. (B) Quantification of the western blot assay. ${ }^{* *}$ P $<0.01$ vs. control (one-way analysis of variance).

expression levels of SATB1 were shown to decrease significantly in the MDA-MB-231 cells following treatment with the different concentrations of baicalein $(10,20$ and $40 \mu \mathrm{M})$ for $48 \mathrm{~h}(\mathrm{P}<0.01)$. The results demonstrated that baicalein reduced the protein expression levels of SATB1 in MDA-MB-231 cells in a dose-dependent manner $(\mathrm{P}<0.05)$. The results of the western blot analysis are shown in Fig. 3 .

\section{Discussion}

Abnormal growth and metastasis of cancer cells are important biological properties of cancer. Invasion and metastasis are the main reasons underlying the cancer morbidity and mortality observed in millions of patients (16). There is an important theoretical and clinical value to screening effective anti-invasive and anti-metastatic drugs with few side-effects, as these drugs improve the curative effect and prognosis of breast cancer, and the quality of life of the patient. Baicalein, the aglycon compound of baicalin, is extracted from the traditional Chinese medicine, Scutellaria baicalensis Georgi. An increasing number of studies have demonstrated that baicalein exhibits potent antitumor properties $(13,16,17)$. The antitumor mechanism of baicalein primarily manifests through the inhibition of tumor cell proliferation, tumor invasion and metastasis. In addition, baicalein offers a synergistic effect to chemotherapy drugs in tumor cells with multidrug resistance $(13,14,18)$. Baicalein has been demonstrated to inhibit a number breast cancer cell lines, including MCF-7, MDA-MB-231, BT549 and 4T-1, through targeting their cell proliferation, invasion and migration ability, distant metastasis and downregulating the protein expression levels of matrix metalloproteinase (MMP)-2, MMP-9 and urinary plasminogen activator $(13,19-21)$. However, the specific antitumor mechanism is yet to be elucidated.

Dickinson et al identified SATB1 from the human cDNA library by using the nuclear matrix-associating DNA sequence, which is located on the $3^{\prime}$ end of the gene enhancer of $\mu$ chain (an immunoglobulin heavy-chain), as a probe (22). SATB1 was revealed to be anchored on chromatin with a unique 'cage-like' structure that was able to provide binding sites for a number of transcription factors. Therefore, SATB1 was hypothesized to play an important role in gene transcription (10). Han et al reported for the first time that SATB1 had abnormally high expression levels in breast cancer (21), while almost no expres- sion was observed in the normal control tissues; thus, SATB1 can be regarded as an independent adverse prognostic factor of breast cancer. SATB1 can promote the growth and metastasis of breast cancer cells, and silencing SATB1 expression in breast cancer MDA-MB-231 cells has been shown to change the invasive phenotype and inhibit tumor growth. Therefore, SATB1 was hypothesized to play a key role in tumor progression. Genomics research found that SATB1 can regulate the expression of $>1,000$ genes that influence the occurrence and development of tumors, including ERBB2, MMP-2, -3 and -9, ABL1 and E-cadherin, and is involved in 61 types of biological activity, such as cell proliferation, apoptosis, DNA synthesis/degradation, electron transport, protein expression and receptor activities (12). SATB1 has been shown to positively correlate with the expression of a variety of biological and genetic markers, including cyclin D1, MMP-2, nuclear factor- $\kappa \mathrm{B}$ and proliferating cell nuclear antigen, while negatively correlating with the expression of APC and BRAF (V600E) $(12,23)$. Therefore, the expression of SATB1 may be used as a novel tool to evaluate the gradation of breast cancer, and SATB1 is expected to become a new therapeutic target in the future.

The MDA-MB-231 human breast cancer cell line has a high level of invasiveness, and a previous study (12) reported that these cells also expressed SATB1 at a high level. In the present study, baicalein was demonstrated to exert a strong inhibitory effect on tumor cell malignant proliferation in a time- and dose-dependent manner. The wound healing assay confirmed that baicalein inhibited the cell migration ability of the breast cancer MDA-MB-231 cells. In addition, small doses of baicalein intervention were shown to downregulate the expression of SATB1 protein, and inhibition was significantly enhanced with increasing concentrations of baicalein $(\mathrm{P}<0.01)$. These results indicate that the downregulation of SATB1 protein expression may be one of the mechanism underlying the inhibitory function of baicalein with regard to the invasion and metastasis of breast cancer cells. However, the present study utilized in vitro experiments only, and a positive control group was not included. Therefore, further research is required, including in vivo experiments.

In conclusion, the present study demonstrated that baicalein strongly suppressed the proliferation and migration ability of MDA-MB-231 cells, possibly through the inhibition of SATB1 protein expression. However, the inhibitory mechanism of 
baicalein on MDA-MB-231 breast cancer cells requires further evaluation in future studies.

\section{Acknowledgements}

The study was supported by grants from the National Natural Science Fund Surface Project (no. 81274136), the Xi'an Jiaotong University Cross Project Fund (no. Xjj2012141) and the talent fund of the Second Affiliated Hospital of Xi'an Jiaotong University (no. RCCGG201105).

\section{References}

1. Friedenreich CM: Physical activity and breast cancer: review of the epidemiologic evidence and biologic mechanisms. Recent Results Cancer Res 188: 125-139, 2011.

2. Ahmad A and Hart IR: Mechanisms of metastasis. Crit Rev Oncol Hematol 26: 163-173, 1997.

3. Sporn MB: The war on cancer. Lancet 347: 1377-1381, 1996.

4. Alvarez JD, Yasui DH, Niida H, Joh T, Loh DY and Kohwi-Shigematsu T: The MAR-binding protein SATB1 orchestrates temporal and spatial expression of multiple genes during T-cell development. Genes Dev 14: 521-535, 2000.

5. Cai S, Han HJ and Kohwi-Shigematsu T: Tissue-specific nuclear architecture and gene expression regulated by SATB1. Nat Genet 34: 42-51, 2003.

6. Mai JC and Ellenbogen RG: SATB1: the convergence of carcinogenesis and chromatin conformation. Neurosurgery 63: N6, 2008.

7. Kohwi-Shigematsu T, Poterlowicz K, Ordinario E, Han HJ, Botchkarev VA and Kohwi Y: Genome organizing function of SATB1 in tumor progression. Semin Cancer Biol 23: 72-79, 2013.

8. Galande S, Purbey PK, Notani D and Kumar PP: The third dimension of gene regulation: organization of dynamic chromatin loopscape by SATB1. Curr Opin Genet Dev 17: 408-414, 2007.

9. Pavan Kumar P, Purbey PK, Sinha CK, Notani D, Limaye A, Jayani RS and Galande S: Phosphorylation of SATB1, a global gene regulator, acts as a molecular switch regulating its transcriptional activity in vivo. Mol Cell 22: 231-243, 2006.

10. Wen J, Huang S, Rogers H, Dickinson LA, Kohwi-Shigematsu T and Noguchi CT: SATB1 family protein expressed during early erythroid differentiation modifies globin gene expression. Blood 105: 3330-3339, 2005.
11. Cai S, Lee CC and Kohwi-Shigematsu T: SATB1 packages densely looped, transcriptionally active chromatin for coordinated expression of cytokine genes. Nat Genet 38: 1278-1288, 2006.

12. Wang L, Ling Y, Chen Y, et al: Flavonoid baicalein suppresses adhesion, migration and invasion of MDA-MB-231 human breast cancer cells. Cancer Lett 297: 42-48, 2010.

13. Chiu YW, Lin TH, Huang WS, et al: Baicalein inhibits the migration and invasive properties of human hepatoma cells. Toxicol Appl Pharmacol 255: 316-326, 2011.

14. Takahashi H, Chen MC, Pham H, et al: Baicalein, a component of Scutellaria baicalensis, induces apoptosis by Mcl-1 down-regulation in human pancreatic cancer cells. Biochim Biophys Acta 1813: 1465-1474, 2011.

15. Lee Y, Yeo H, Liu SH, Jiang Z, Savizky RM, Austin DJ and Cheng YC: Increased anti-P-glycoprotein activity of baicalein by alkylation on the A ring. J Med Chem 47: 5555-5566, 2004.

16. Lee WJ, Wu LF, Chen WK, Wang CJ and Tseng TH: Inhibitory effect of luteolin on hepatocyte growth factor/scatter factor-induced HepG 2 cell invasion involving both MAPK/ERKS and PI3K-Akt pathways. Chem Biol Interact 160: 123-133, 2006.

17. Po LS, Chen ZY, Tsang DS and Leung LK: Baicalein and genistein display differential actions on estrogen receptor (ER) transactivation and apoptosis in MCF-7 cells. Cancer Lett 187: 33-40, 2002.

18. Wu B, Li J, Huang D, et al: Baicalein mediates inhibition of migration and invasiveness of skin carcinoma through Ezrin in A431 cells. BMC Cancer 11: 527, 2011.

19. Huang S, New L, Pan Z, Han J and Nemerow GR: Urokinase plasminogen activator/urokinase-specific surface receptor expression and matrix invasion by breast cancer cells requires constitutive p38alpha mitogen-activated protein kinase activity. J Biol Chem 275: 12266-12272, 2000.

20. Gunther S, Ruhe C, Derikito MG, Böse G, Sauer H and Wartenberg M: Polyphenols prevent cell shedding from mouse mammary cancer spheroids and inhibit cancer cell invasion in confrontation cultures derived from embryonic stem cells. Cancer Lett 250: 25-35, 2007.

21. Han HJ, Russo J, Kohwi Y and Kohwi-Shigematsu T: SATB1 reprogrammes gene expression to promote breast tumour growth and metastasis. Nature 452: 187-193, 2008.

22. Dickinson LA, Joh T, Kohwi Y and Kohwi-Shigematsu T: A tissue-specific MAR/SAR DNA-binding protein with unusual binding site recognition. Cell 70: 631-645, 1992.

23. Zhang J, Zhang B, Zhang X, et al: SATB1 expression is associated with biologic behavior in colorectal carcinoma in vitro and in vivo. PLoS One 8: e47902, 2013. 